\title{
Primary ciliary dyskinesia ciliated airway cells show increased susceptibility to Haemophilus influenzae biofilm formation
}

\begin{abstract}
Woolf T. Walker 1,2,3,7, Claire L. Jackson 1,2,3,7, Raymond N. Allan ${ }^{3,4}$, Samuel A. Collins (1) ${ }^{1,2,3}$, Michael J. Kelso ${ }^{5}$, Ardeshir Rineh ${ }^{5}$, Nageshwar R. Yepuri ${ }^{5}$, Ben Nicholas ${ }^{2,3}$, Laurie Lau ${ }^{2,3}$, David Johnston ${ }^{2,3}$, Peter Lackie ${ }^{1,2,3}$, Saul N. Faust ${ }^{2,3,4}$, Jane S.A. Lucas (1) ${ }^{1,2,3,8}$ and Luanne Hall-Stoodley ${ }^{2,3,4,6,8}$

Affiliations: ${ }^{1}$ Primary Ciliary Dyskinesia Centre, University Hospital Southampton NHS Foundation Trust, Southampton, UK. ${ }^{2} \mathrm{NIHR}$ Southampton Biomedical Research Centre, University of Southampton and University Hospital Southampton NHS Foundation Trust, Southampton, UK. ${ }^{3}$ Academic Unit of Clinical and Experimental Sciences, University of Southampton Faculty of Medicine, Southampton, UK. ${ }^{4}$ Southampton NIHR Wellcome Trust Clinical Research Facility, University of Southampton and University Hospital Southampton NHS Foundation Trust, Southampton, UK. ${ }^{5}$ Illawarra Health and Medical Research Institute, School of Chemistry, University of Wollongong. Wollongong, Australia. ${ }^{6}$ Dept of Microbial Infection and Immunity, Centre for Microbial Interface Biology, College of Medicine, The Ohio State University, Columbus, OH, USA. "Both authors contributed equally. ${ }^{8}$ Both authors contributed equally.
\end{abstract}

Correspondence: Luanne Hall-Stoodley, The Ohio State University College of Medicine - Microbial Infection and Immunity, 714 BRT 460 West 12th Avenue, Columbus, OH 43210, USA.

E-mail: Luanne.Hall-Stoodleydosumc.edu

@ERSpublications

Ex vivo study shows PCD airways risk NTHi biofilm colonisation; novel NO drug overcomes antibiotic tolerance http://ow.ly/GrXC30e4Tqv

Cite this article as: Walker WT, Jackson CL, Allan RN, et al. Primary ciliary dyskinesia ciliated airway cells show increased susceptibility to Haemophilus influenzae biofilm formation. Eur Respir J 2017; 50: 1700612 [https://doi.org/10.1183/13993003.00612-2017]

ABSTRACT Non-typeable Haemophilus influenzae (NTHi) is the most common pathogen in primary ciliary dyskinesia (PCD) patients. We hypothesised that abnormal ciliary motility and low airway nitric oxide (NO) levels on airway epithelial cells from PCD patients might be permissive for NTHi colonisation and biofilm development.

We used a primary epithelial cell co-culture model to investigate NTHi infection. Primary airway epithelial cells from PCD and non-PCD patients were differentiated to ciliation using an air-liquid interface culture and then co-cultured with NTHi.

NTHi adherence was greater on PCD epithelial cells compared to non-PCD cells $(p<0.05)$ and the distribution of NTHi on PCD epithelium showed more aggregated NTHi in biofilms $(\mathrm{p}<0.001)$. Apart from defective ciliary motility, PCD cells did not significantly differ from non-PCD epithelial cells in the degree of ciliation and epithelial integrity or in cytokine, LL-37 and NO production. Treatment of PCD epithelia using exogenous NO and antibiotic significantly reduced NTHi viability in biofilms compared with antibiotic treatment alone.

Impaired ciliary function was the primary defect in PCD airway epithelium underlying susceptibility to NTHi biofilm development compared with non-PCD epithelium. Although NO responses were similar, use of targeted NO with antibiotics enhanced killing of NTHi in biofilms, suggesting a novel therapeutic approach. 


\section{Introduction}

Motile cilia in the airway contribute to mucociliary clearance (MCC), which is fundamental for protecting the host from respiratory infection. Primary ciliary dyskinesia (PCD) is a genetically heterogeneous condition characterised by abnormal ciliary function with compromised MCC. Patients are characterised by recurrent and chronic sinopulmonary infection, chronic otitis media $(\mathrm{OM})$ and progressive suppurative lung disease from a young age [1].

Non-typeable Haemophilus influenzae (NTHi) is a Gram-negative coccobacillus and, with pneumococcus, the most common bacterial pathogen isolated from the upper and lower respiratory tract of paediatric patients with PCD [2] and chronic OM [3]. NTHi biofilms are present in vivo [4, 5] and ex vivo on adenoid epithelium, or middle ear epithelial biopsies from children with OM [6, 7].

Biofilms are self-organising aggregates of bacteria enveloped in an exopolymeric matrix associated with the respiratory mucosal surface $[8-10]$ and are present in chronic upper and lower respiratory tract infections in OM [6], cystic fibrosis (CF) [11], chronic rhinosinusitis [12] and non-CF bronchiectasis [13]. Biofilm development contributes to the pathophysiology of chronic suppurative respiratory diseases by increasing bacterial recalcitrance to antibiotic therapy and host innate immune responses [14, 15]. Although the effects of abnormal ciliary function on respiratory infections have been studied in animal models of bacteria-virus co-infection [16,17], bacterial infection has not been widely studied in PCD.

For reasons that are not understood, patients with PCD have low airway nitric oxide (NO) levels compared with non-PCD patients $[18,19]$. NO, produced by host epithelial cells and innate immune cells, can be low in response to infection by some respiratory bacteria $[20,21]$. We previously observed no difference in the mean NO levels of uninfected primary cultured PCD and non-PCD epithelia, consistent with baseline NO levels reported by others [20, 22]. However, we found PCD epithelial cells significantly increased NO production in response to either stimulation with pro-inflammatory cytokines or to NTHi infection, similar to non-PCD cells [22]. Given the susceptibility of children with PCD to airway infection with NTHi, we hypothesised that epithelial cells from patients with PCD might be permissive for increased NTHi adherence and subsequent biofilm development. To address this hypothesis, we developed a human primary co-culture model using ciliated PCD or non-PCD airway epithelial cells, differentiated at an airliquid interface (ALI) and infected with a PCD clinical NTHi isolate. In the present study, we used the ALI-epithelial cell NTHi co-culture model [22] to more extensively investigate: 1) airway cell responses to $\mathrm{NTHi}$, including NO production, and 2) the ability of a biofilm-targeted NO donor to augment antibiotic treatment of NTHi.

\section{Materials and methods \\ Study participants}

We recruited 15 patients with a diagnosis of PCD, according to the 2009 European guidelines [23], 10 healthy volunteers and nine patients with respiratory symptoms not caused by PCD (table S1). We have previously published details of the diagnostic methods undertaken at our national PCD Centre [24]. The study gained UK National Health Service Research Ethics approval (06/Q1702/109 and 08/H0502/126) and we obtained written, informed consent.

\section{Culture and biofilm development by clinical NTHi isolates from PCD patients}

NTHi isolates from four paediatric PCD patients (HI1-HI4) were subcultured from frozen stocks [25] and verified by Gram stain, V and X diagnostic disc testing, and polymerase chain reaction [6]. NTHi was cultured in brain-heart infusion medium supplemented with haemin and nicotinamide adenine dinucleotide $(\mathrm{sBHI})$ at $37^{\circ} \mathrm{C}$ to $\mathrm{OD}_{600} 0.1\left(\sim 2 \times 10^{8} \mathrm{CFU} \cdot \mathrm{mL}^{-1}\right.$ for all strains tested) [25]. Biofilm growth was determined in vitro in triplicate experiments by crystal violet $(\mathrm{CV})$ staining and colony forming unit (CFU) counts on chocolate blood agar (CBA) $[26,27]$ and by scanning electron microscopy and confocal microscopy using fluorescence in situ hybridisation (FISH) [7, 28].

This article has supplementary material available from erj.ersjournals.com

Received: March 232017 | Accepted after revision: June 042017

Support statement: The National PCD Centre at UHS is commissioned and funded by NHS England. PCD research in Southampton is supported by NIHR Southampton Respiratory Biomedical Research Unit and the Southampton NIHR Wellcome Trust Clinical Research Facility. W.T. Walker, C.L. Jackson, S.A. Collins, J.S.A. Lucas and L. Hall-Stoodley are participants in COST Action BEAT-PCD (BM1407). M.J. Kelso acknowledges funding from the Australian Cystic Fibrosis Research Trust (ACFRT). Funding information for this article has been deposited with the Crossref Funder Registry.

Conflict of interest: Disclosures can be found alongside this article at erj.ersjournals.com 
Epithelial cell and NTHi co-culture model

Detailed information regarding epithelial cell recovery and subsequent analysis is available in the supplementary material and has been published elsewhere. Briefly, primary human nasal epithelial cells were cultured at an ALI until differentiated and ciliated [29, 30]. We used ALI-cultured cells within 1 month. NTHi was co-cultured on the apical surface of epithelial cells from five PCD and nine non-PCD subjects and compared with five non-infected non-PCD controls. We inoculated approximately $1 \times 10^{8} \mathrm{CFU} \cdot \mathrm{mL}^{-1}$ in $500 \mu \mathrm{L}$ of antibiotic-free ALI medium (multiplicity of infection 100) onto ALI-cultured cells on transwell membranes. Co-cultures were incubated at $37^{\circ} \mathrm{C} / 5 \% \mathrm{CO}_{2}$ for $72 \mathrm{~h}$ with daily medium changes and washed with Hanks' buffered salt solution (HBSS) to remove non-adherent bacteria prior to analyses [25].

Transepithelial electrical resistance (TEER) was measured daily and background subtracted to confirm epithelial integrity (EVOM 2 epithelial voltohmmeter, World Precision Instruments, Sarasota, FL, USA) [31]. Ciliary beat frequency (CBF) and the percentage area of motile to non-motile cilia on ALI-cultured cells before and during NTHi co-culture was measured by whole-field fast Fourier transform analysis (ImageJ https://imagej.net/ with an in-house written plugin) of high-speed video microscopy videos. Briefly, samples were imaged at $37^{\circ} \mathrm{C}$ using an inverted Olympus IX71 microscope with $\times 40$ objective lens (Olympus, Southend-on-Sea, UK) $(1$ field $=90 \times 95 \mu \mathrm{m})$ and a PC2 Photron FASTCAM high-speed video recorded ciliary movement at 250 frames per second [29].

\section{Characterisation of NTHi on ciliated airway cells}

Bacteria and epithelial cells were scraped from transwell membranes into sBHI, serially diluted, plated onto $\mathrm{CBA}$ and incubated at $37^{\circ} \mathrm{C} / 5 \% \mathrm{CO}_{2}$ before $\mathrm{CFU}$ enumeration (triplicate experiments). We also identified NTHi on ALI culture samples using confocal microscopy by FISH with the Hinf 16S probe [6, 7] and $\beta$-tubulin in cilia co-labelled by immunofluorescence [32]. Consecutive fields across the membrane diameter were imaged using an SP5 confocal laser scanning microscope (Leica Microsystems, Milton Keynes, UK) and NTHi volume per field was calculated by Volocity 3D image analysis software (version 6.0.1; PerkinElmer, Coventry, UK). Selected transwell membranes were removed and processed and imaged using an FEI Quanta 200 scanning electron microscope (FEI, Eindhoven, the Netherlands) [25, 28].

\section{Cytokine and LL-37 responses to NTHi infection}

Basolateral medium from ALI cultures was frozen before inoculation and then daily following co-culture with NTHi. We measured fibroblast growth factor- $\beta$ (FGF- $\beta$ ), granulocyte colony-stimulating factor (G-CSF), granulocyte-macrophage colony-stimulating factor (GM-CSF), interleukin-6 (IL-6), interleukin-1 receptor antagonist (IL-1Ra), monocyte chemotactic protein-1 (MCP-1), macrophage inflammatory protein-1 $\alpha$ (MIP-1 $\alpha)$, tumour necrosis factor- $\alpha$ (TNF- $\alpha)$ and vascular endothelial growth factor (VEGF) by fluorokine human multianalyte profiling (MAP) assay with Bio-Plex 200 Analyser detection (R\&D systems, Abingdon, UK) in supernatants (50 $\mu \mathrm{L}$ per 96 wells in duplicate) (supplementary material) [33]. Interleukin-8 was measured separately by human IL-8 DuoSet ELISA kit (DY208, R\&D systems) and horseradish peroxidase (HRP) substrate was detected by a ThermoMax Microplate Reader (Molecular Devices, Sunnyvale, CA, USA) as per the manufacturer's instructions.

Apical supernatants (100 $\mu \mathrm{L}$ PBS) from ALI-cultured cells were frozen before co-culture and then daily following NTHi inoculation. Samples were dotted onto nitrocellulose membranes and immunolabelled overnight $\left(4^{\circ} \mathrm{C}\right)$ with 1:1000 rabbit anti-LL-37 antibody (AB64892; Abcam, Cambridge, UK) followed by 1:10000 HRP conjugated goat anti-rabbit secondary antibody. After washes, Immuno-star Luminol chemiluminescent substrate was detected using a VersaDoc Imager and quantified by Quantity One v4.6.9 software (Bio-Rad Laboratories, Watford, UK). LL-37 concentrations (duplicate experiments) were compared with a standard curve from serially diluted recombinant LL-37 peptide (AB140725; Abcam).

\section{NO production by airway epithelial cells and treatment with exogenous NO}

Transwell membranes of live ALI-cultured epithelial cells were treated with $10 \mu \mathrm{M}$ 4-amino-5-methylamino-2', $7^{\prime}$-difluorofluorescein diacetate (DAF-FM) to assess the presence of NO [34]. Immunofluorescence labelling was used to localise NO synthase (NOS) (neuronal (nNOS), inducible (iNOS) and endothelial (eNOS) isoenzymes in fixed epithelial cell cultures [32] using confocal microscopy.

ALI-cultured epithelial cell-NTHi co-cultures $(72 \mathrm{~h})$ were washed with HBSS to remove non-adherent bacteria [25] and then treated with either $4 \mathrm{mg} \cdot \mathrm{mL}^{-1}$ azithromycin, $50 \mu \mathrm{M}$ cephalosporin-3'-diazeniumdiolate NO donor prodrug PYRRO-C3D, or with a combination of both azithromycin and PYRRO-C3D for $2 \mathrm{~h}$. All treatments were prepared in HBSS with 1\% DMSO (also used as the untreated control), and NTHi CFUs were enumerated on CBA as above $(n=4)$ [25]. 


\section{Statistical methods}

The t-test and Mann-Whitney U-test were performed using Graphpad Prism software (version 6.0a, 2012, Graphpad Software, Inc.). The Sidak-Bonferroni method was used to adapt p-values for multiple comparison t-tests. $\mathrm{p}<0.05$ demonstrated statistical significance.

\section{Results}

\section{Study participants}

Demographic and clinical details of the 15 PCD and 19 non-PCD participants are presented in table 1. All PCD patients exhibited abnormal ciliary function (table S1).

\section{Biofilm development by clinical NTHi isolates from PCD patients}

We assessed the ability of four PCD NTHi isolates to form biofilms by standard assays (CV, CFU counts) and by scanning electron microscopy and confocal microscopy using the Hinf-specific 16S FISH probe (supplementary material) (figure 1). Clinical isolates (HI3 and HI4), from children who repeatedly cultured NTHi over 4 years, demonstrated biofilm development with significantly greater CV staining of exopolymeric matrix compared with isolates from children culturing NTHi for the first time (HI1 and HI2) (figure 1a). However, CFU counts were not significantly different between isolates (figure 1b). Isolate HI4 was used for future experiments.

\section{Epithelial cell and NTHi co-culture}

NTHi adhered to ciliated airway epithelial cells from both PCD and non-PCD patients. At $72 \mathrm{~h}$, however, there was significantly more viable NTHi $3.8 \times 10^{6} \mathrm{CFUs}\left( \pm 7.5 \times 10^{5}\right)$ on PCD epithelia compared with $2.0 \times 10^{6}$ CFUs $\left( \pm 4.8 \times 10^{5}\right)$ on non-PCD epithelia $(\mathrm{p}<0.05)$ (figure $\left.2 \mathrm{a}\right)$, in agreement with scanning electron microscopy data (figure $2 \mathrm{c}, \mathrm{d}$ ). PCD cells harboured discrete adherent aggregates of bacterial cells (biofilms) (figure 2c) in contrast to the singularly distributed NTHi bacterial cells on non-PCD epithelial cells (figure $2 \mathrm{~d}$ ).

We imaged co-cultures by confocal microscopy using a combination of FISH to label NTHi and an anti- $\beta$-tubulin antibody to label cilia. Orthogonal section image analysis of confocal microscope Z-stacks showed that the mean \pm SEM NTHi biomass volume (per field of view) was four times greater on PCD ALI-cultured epithelia $\left(710 \pm 150 \mu \mathrm{m}^{3}\right)$ than non-PCD primary cell ALI cultures $\left(160 \pm 20 \mu \mathrm{m}^{3}\right)(\mathrm{p}<0.001)$ (figure 2b). Aggregated NTHi were evident in biofilms on PCD primary cell epithelia that had dysfunctional or static cilia (figure 2e). In contrast there was less NTHi labelling on non-PCD epithelia with normally functioning cilia (figure $2 \mathrm{f}$ ).

PCD cells showed either static $(n=4)$ or slow dyskinetic $(n=1)$ cilia. The CBF of non-PCD epithelial cells remained within normal ranges $(11-20 \mathrm{~Hz})$ throughout the $72 \mathrm{~h}$ of NTHi co-culture, indicating that NTHi did not impair ciliary motility (figure 3a). Ciliation of ALI-cultured epithelial cells was similar in PCD and non-PCD, as observed by light microscopy and scanning electron microscopy (figure $3 \mathrm{~b}$ ). The percentage area of motile cilia per field of view on non-PCD ALI-cultured cells did not change during the co-culture period on non-PCD airway cells (figure 3c). TEER was used to measure epithelial cell barrier function before and after apical NTHi inoculation onto ciliated ALI-cultured primary airway cells. The TEER plateau reached prior to NTHi infection was the same for PCD and non-PCD cells and was maintained for $72 \mathrm{~h}$ in co-culture, demonstrating epithelial integrity ( $\mathrm{p}$-values ranged from 0.35 to 0.93 ) (figure $3 \mathrm{~d}$ ).

TABLE 1 Demographics of the primary ciliary dyskinesia (PCD), respiratory control and healthy participants

\begin{tabular}{lccc} 
& PCD & \multicolumn{2}{c}{ Non-PCD } \\
\cline { 3 - 3 } & & Respiratory control & Healthy \\
\hline Subjects $\mathbf{n}$ & 15 & 9 & 10 \\
Male $\mathbf{n}(\%)$ & $10(66.7)$ & $2.9(4.8)$ & $3(30)$ \\
Median age in years (IQR) & $16.85(19.89)$ & $8(88.9)$ & $32.55(14.96)$ \\
Pulmonary symptoms $\mathbf{n}(\%)$ & $15(100)$ & $7(77.8)$ & 0 \\
Rhinosinusitis $\mathbf{n}(\%)$ & $14(93.3)$ & $1(11.1)^{\#}$ & 0 \\
Situs abnormality $\mathbf{n}(\%)$ & $9(60)$ & & 0 \\
\hline \#: normal diagnostic tests (table S1, participant 018). & & \\
\hline
\end{tabular}



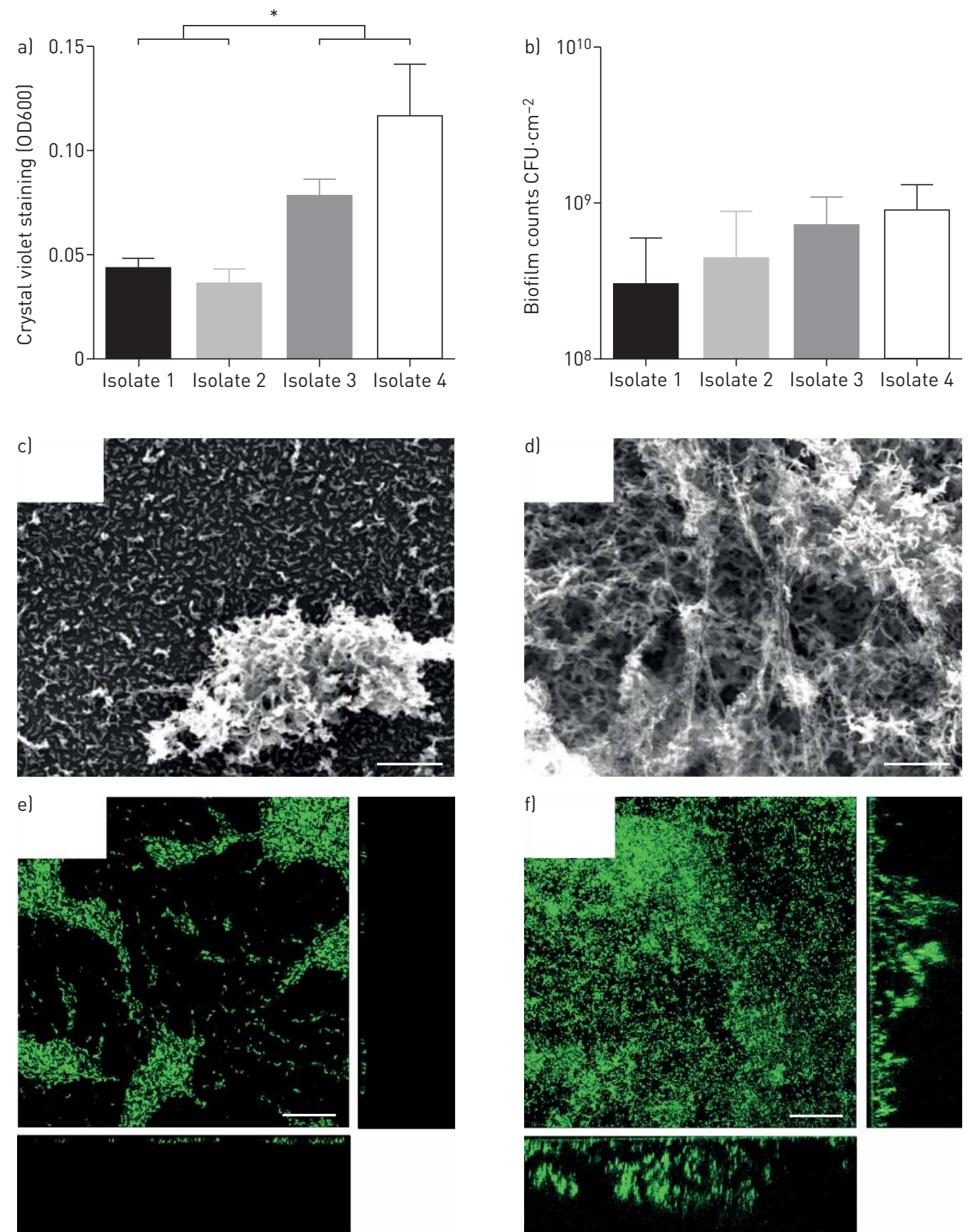

FIGURE 1 Biofilm formation by primary ciliary dyskinesia (PCD) non-typeable Haemophilus influenzae (NTHi) isolates (HI1-4). Bar charts show the mean \pm SEM optical densities (OD600) of crystal violet staining (a) and mean \pm SEM log biofilm colony forming unit counts $\left(C F U \cdot \mathrm{cm}^{-2}\right)(\mathrm{b})$ for PCD-clinical NTHi isolates (HI1-4) grown for $72 \mathrm{~h}$ $\left(^{*}: p<0.05\right)$. Scanning electron micrographs $(\times 4000)(c, d)$ highlight bacterial aggregates and exopolymeric matrix for HI1 and HI4. Confocal micrographs (e,f) depict representative maximum projections (of Z-stacks) and orthogonal sections ( $\mathrm{x} / \mathrm{z}$ right and $\mathrm{y} / \mathrm{z}$ bottom images) of $72 \mathrm{~h} \mathrm{HI1}$ and HI4 biofilms hybridised with the CY3-labelled $H$. influenzae-specific fluorescence in situ hybridisation probe (green). Scale bars=10 $\mu \mathrm{m}$ (c, d); $40 \mu \mathrm{m}$ (e, f).

Cytokine and LL-37 responses to NTHi infection

The mean \pm SEM concentration of cationic LL-37 peptide secreted from epithelial cell ALI-cultures peaked $24 \mathrm{~h}$ following NTHi inoculation, where an 8.8 \pm 2.9 -fold increase in PCD and 3.8 \pm 0.6 -fold increase in non-PCD samples was observed, but values remained within group variation at baseline and after inoculation (figure $4 \mathrm{a}, \mathrm{b}$ ). The levels of FGF- $\beta$, G-CSF, GM-CSF, VEGF or IL-1Ra, IL-6, IL-8, MCP-1, MIP- $1 \alpha$ and TNF- $\alpha$ in basolateral medium from PCD or non-PCD cells, sampled before or daily during $72 \mathrm{~h}$ of NTHi co-culture (figure 4c) did not significantly differ, indicating that PCD airway cells produced 

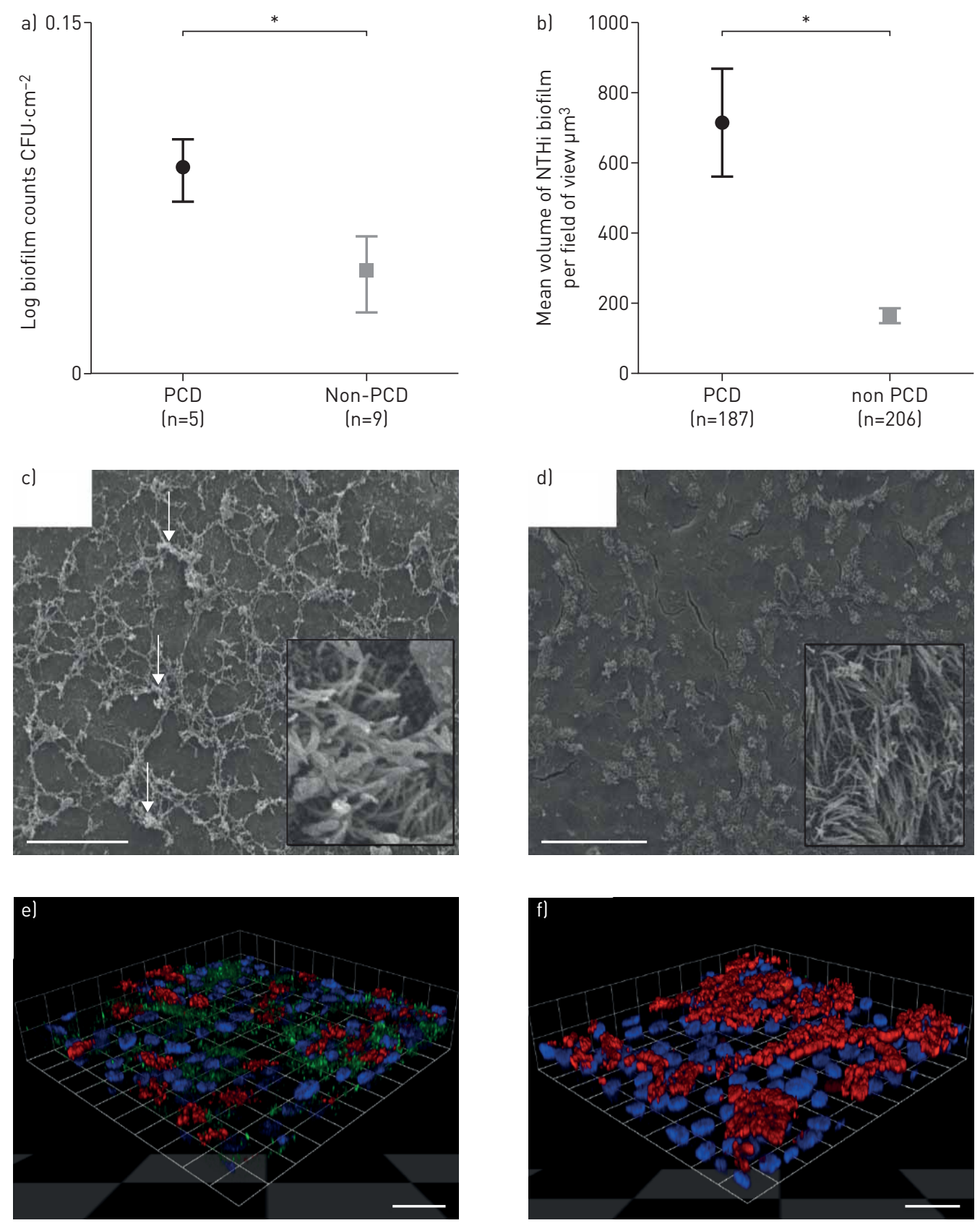

FIGURE 2 Characterisation of non-typeable Haemophilus influenzae (NTHi) co-cultured with air-liquid interface (ALI) differentiated and ciliated airway epithelial cells. a) The mean \pm SEM log biofilm CFU. $\mathrm{cm}^{-2}$ after $72 \mathrm{~h}$ of NTHi co-culture on ALI ciliated primary ciliary dyskinesia (PCD) ( $n=5)$, and non-PCD ( $n=9$ ) epithelial cells (*: $p<0.05)$. Representative scanning electron micrographs $(\times 4000)(c, d)$ demonstrate increased bacterial aggregates and exopolymeric matrix formation (arrows) on PCD ALI-cultured ciliated epithelial cells in contrast to non-PCD cells. NTHi co-cultured on ALI ciliated PCD and non-PCD epithelial cells were labelled for confocal analysis. Bacterial cells were labelled with the CY3-H. influenzae-specific fluorescence in situ hybridisation (FISH) probe (green), cilia were labelled with anti- $\beta$-tubulin antibody (red) and nuclei were counterstained with SYTOX blue (blue). b) The mean \pm SEM biofilm volume (FISH probe labelling) per field of view (determined by Volocity software analysis of confocal microscopy z-stacks) on PCD versus non-PCD ciliated ALI cultures (*: p<0.05). Confocal micrographs $(e, f)$ are represented as three-dimensional opacity views of ciliated PCD (e), and non-PCD (f) cells co-cultured for $72 \mathrm{~h}$ with NTHi. Scale bars $=20 \mu \mathrm{m}$.

cytokines and chemokines similarly to non-PCD cells. These data indicate that PCD and non-PCD epithelial cells produce similar defence responses to NTHi.

NO production by airway epithelial cells and treatment with exogenous NO

Airway levels of NO are extremely low in patients with PCD [18, 19]. However, we found using immunofluorescence labelling of epithelial cells that the nNOS, iNOS and eNOS isoenzymes are present in 

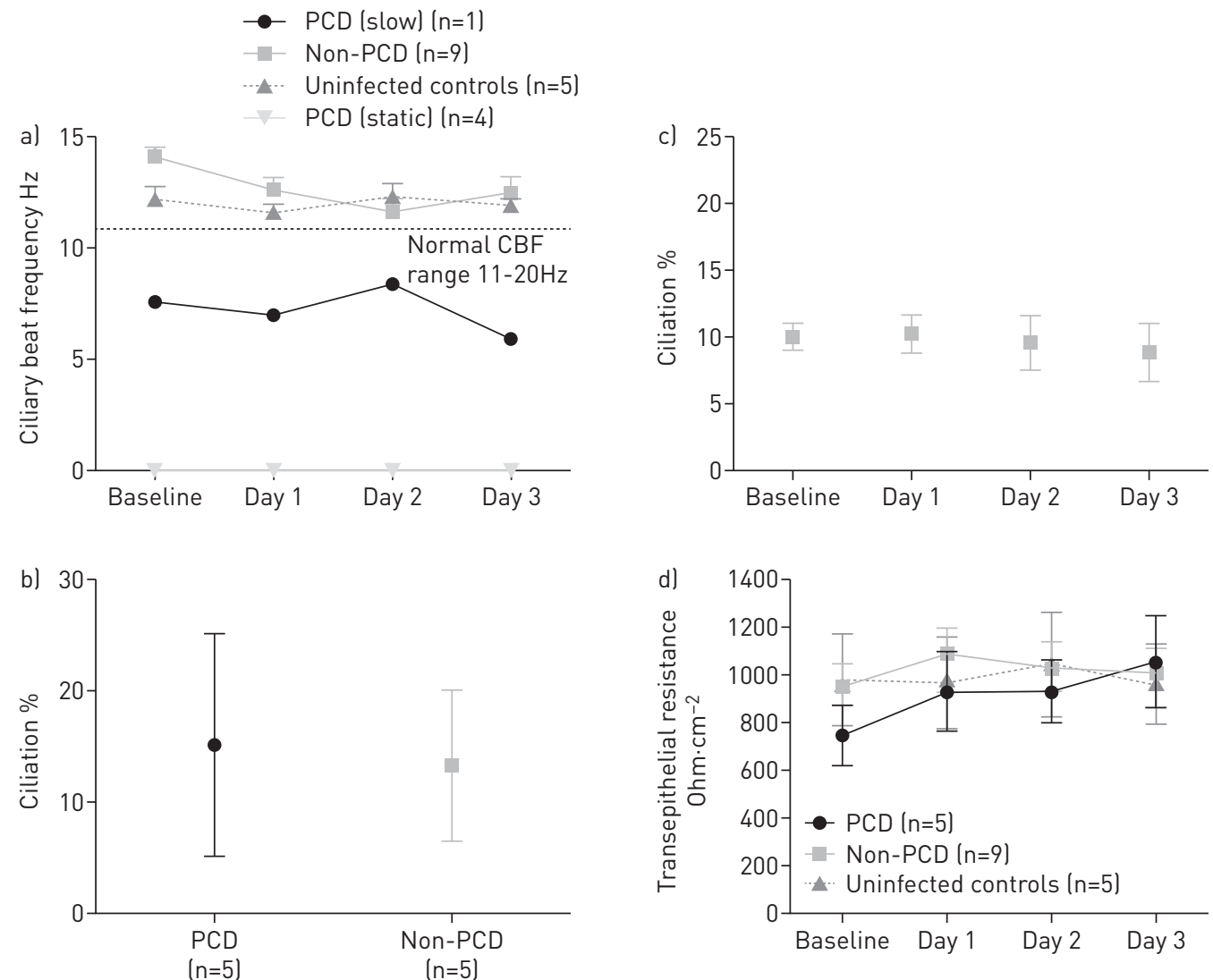

FIGURE 3 Comparison of percent ciliation, ciliary function and trans-epithelial electrical resistance (TEER) in primary ciliary dyskinesia (PCD) and non-PCD air-liquid interface (ALI)-cultured airway epithelial cells, and characterisation of the effect of non-typeable Haemophilus influenzae (NTHi) co-culture on cilia. a) The mean \pm SEM ciliary beat frequency (CBF) (in Hz) of PCD ( $n=4$ static cilia and $n=1$ slow dyskinetic cilia) and non-PCD $(\mathrm{n}=9)$ measured before (baseline) and daily during $72 \mathrm{~h}$ of NTHi co-culture, compared to uninfected non-PCD controls $(n=5)$. The normal CBF range is $11-20 \mathrm{~Hz}$ at the Southampton PCD Centre and infection did not modify CBF in any group. b) The mean \pm SEM percentage ciliation corresponding to coverage of $\beta$-tubulin immunolabelled cilia on differentiated ALI cultures. 10 randomly selected microscopic fields of view were compared per sample and there was no difference in the percentage ciliation between PCD ( $n=5)$ and non-PCD $(n=5)$ samples. c) The mean \pm SEM percentage ciliation calculated from whole field fast Fourier transform analysis of CBF where the ratio of motile to not-motile pixels (15360 data points per total field of view from a video recorded at a resolution of $512 \times 480$; analysis box size $4 \times 4$ pixels). The mean \pm SEM percentage ciliation of non-PCD ( $n=8)$ ALI-cultured cells before (baseline) and daily after NTHi co-culture did not change over $72 \mathrm{~h}$. d) The mean \pm SEM TEER in ohms $\cdot \mathrm{cm}^{-2}$ of NTHi-infected PCD (n=5) and non-PCD ( $\mathrm{n}=9$ ), measured before (baseline) and daily during co-culture for $72 \mathrm{~h}$ was compared to uninfected non-PCD controls $(n=5)$. TEER remained stable and was not significantly different between groups.

both ciliated PCD and non-PCD cells (figure 5). Additionally, we detected NO in PCD and non-PCD ALI culture samples using DAF-FM, which strongly fluoresces in the presence of NO (figure 5).

We have previously shown that NO modulates NTHi metabolism in vitro [25]. Therefore, we tested if exogenous NO treatment impacted NTHi growing on ciliated PCD epithelial cells. Epithelial cell-NTHi co-cultures were treated with the cephalosporin-3'-diazeniumdiolate NO donor prodrug, PYRRO-C3D, which releases NO following activation by $\beta$-lactamases [25]. We found that $4 \mathrm{mg} \cdot \mathrm{mL}^{-1}$ azithromycin treatment, which is in excess of the planktonic minimum inhibitory concentration $\left(1 \mu \mathrm{g} \cdot \mathrm{mL}^{-1}\right)$, failed to kill biofilm NTHi on PCD epithelial cells. In contrast, when PYRRO-C3D was used alongside azithromycin, NTHi viability decreased nearly 3 logs (figure 6). These results suggest that NTHi in biofilms on PCD ciliated epithelial cells are tolerant of antibiotic treatment and that tolerance was abrogated by $\beta$-lactamase-mediated delivery of NO from the prodrug to NTHi.

\section{Discussion}

Our principal finding was that NTHi formed adherent biofilms on ALI-cultured PCD ciliated airway cells significantly more than on non-PCD controls over $72 \mathrm{~h}$ of co-culture, with the only attributable difference being ciliary dysfunction of PCD samples. Since host-pathogen interactions have not been widely studied 

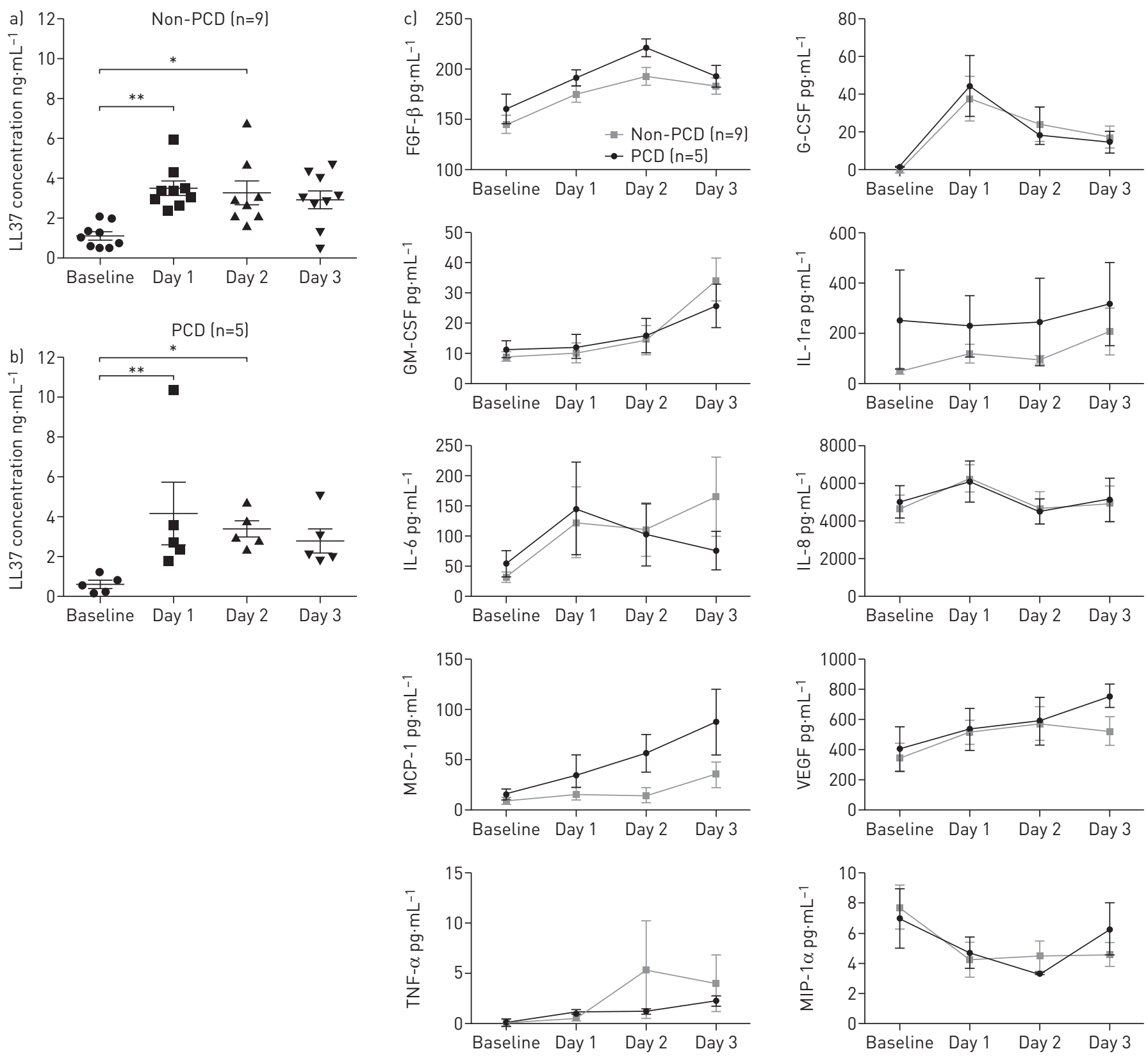

FIGURE 4 Primary ciliary dyskinesia (PCD) and non-PCD epithelial cell LL-37 and cytokine responses to non-typeable Haemophilus influenzae (NTHi) infection. Dot and whisker plots in (a) and (b) demonstrate the median (interquartile range) concentration of cationic antimicrobial peptide, cathelicidin LL-37 (ng. $\mathrm{mL}^{-1}$ ), measured in the apical supernatants of air-liquid interface (ALI)-cultured epithelial cells from non-PCD (a) and PCD (b) patients, before (baseline) or daily after NTHi co-culture for $72 \mathrm{~h}$. (c) The mean \pm SEM concentrations (pg. $\mathrm{mL}^{-1}$ ) of $10 \mathrm{cytokines:} \mathrm{basic} \mathrm{fibroblast}$ growth factor- $\beta$ (FGF- $\beta$ ), granulocyte-colony stimulating factor (G-CSF), granulocyte macrophage-colony stimulating factor (GM-CSF), interleukin-6 (IL-6), interleukin-8 (IL-8), interleukin-1 receptor antagonist (IL-1ra), monocyte chemotactic protein-1 (MCP-1), macrophage inflammatory protein-1 $\alpha$ (MIP-1 $\alpha$ ), tumour necrosis factor $-\alpha$ (TNF- $\alpha$ ) and vascular endothelial growth factor (VEGF) in the basolateral medium of PCD ( $n=5)$ and non-PCD ( $n=9$ ) epithelial cell ALI cultures before (baseline) and daily after NTHi co-culture for 72 h. *: $p<0.05,{ }^{* *}: p<0.01$.

in the context of PCD, these results are novel. NTHi is prevalent in PCD and other diseases in children associated with impaired mucociliary clearance, such as CF [35] and chronic OM [36]. NTHi biofilm development features in the pathophysiology of chronic or recurrent respiratory infections in both CF and chronic OM $[6,4,13]$, but little is known about NTHi infection in PCD. We hypothesised that abnormal ciliary motility on airway epithelial cells from PCD patients might be permissive for NTHi colonisation and biofilm development. To our knowledge, our polarised airway epithelial ALI culture infection model is the first to examine the effects of NTHi on primary ciliated airway cells from PCD patients and non-PCD donors. Biofilm development was previously demonstrated with NTHi isolates from young CF patients 

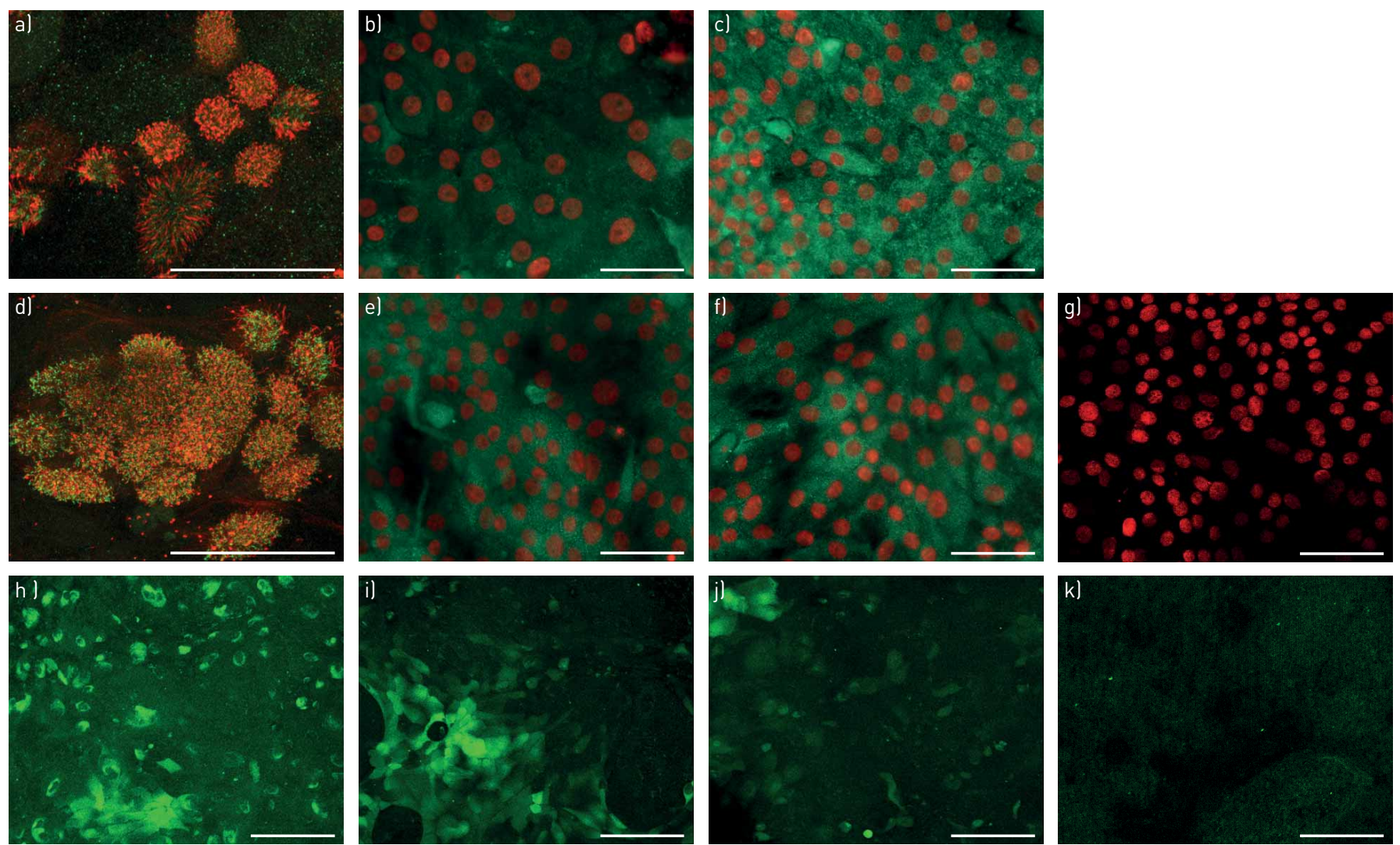

FIGURE 5 Nitric oxide synthase (NOS) isoenzymes and nitric oxide (NO) were compared between ciliated primary ciliary dyskinesia (PCD) and non-PCD airway epithelial cells cultured at air-liquid interface (ALI). Representative micrographs of ALI-differentiated epithelial cells show fluorescent labelling with antibodies to neuronal NOS (a, d), inducible NOS (b, e) and endothelial NOS (c, f) and an Alexafluor488 conjugated secondary antibody (green) in non-PCD $(a-c)$ and PCD $(d-f)$ subjects ( $n=3$ repeats per group). As a negative control primary antibodies were omitted and confirmed that there was no non-specific secondary antibody labelling in non-PCD ALI-cultured epithelium (g). Representative micrographs show the presence of NO by 4-amino-5-methylamino-2',7'-difluorofluorescein diacetate (DAF-FM) labelling (green) in one non-PCD (h) and in two PCD $(i, j)$ subjects ( $n=4$ non-PCD and $n=3$ PCD subjects were tested in total). There was no appreciable difference in NOS isoenzyme or DAF-FM labelling between non-PCD and PCD ALI-cultures. Very low background autofluorescence can be seen in an untreated human bronchial epithelial cell line (16HBE) negative control ( $k$ ). Cell nuclei were stained with Hoechst (red) (b, c, e-g) and cilia were co-labelled with an anti- $\beta$-tubulin antibody (shown in red) $(a, d)$. Merged maximum projections from confocal $(a, d, h-k)$ or epifluorescent (b, $c$, e-g) microscope images using $100 \times$ or $40 \times$ objectives ( $40 \mu \mathrm{m}$ scale bars).

using Calu-3 cell co-cultures [11]. However, because Calu-3 cells do not ciliate, this model did not assess the role of cilia in NTHi adherence and biofilm development [37].

We compared PCD and non-PCD epithelial cell responses, including secreted inflammatory and antimicrobial proteins and epithelial integrity, ciliation and ciliary function following NTHi co-culture. Cytokine production, including pro-inflammatory cytokines and chemokines involved in leukocyte recruitment, and secreted levels of the antimicrobial peptide LL-37, were commensurable between PCD and non-PCD co-cultures. Epithelial integrity was also comparable in airway epithelial cells co-cultured with NTHi. Although all PCD ALI-cultured epithelial cells had dysfunctional ciliary movement, in contrast to the normally functioning non-PCD epithelial cells (table S1), the total ciliation pre-infection was similar, as demonstrated by scanning electron microscopy and immunofluorescent labelling of $\beta$-tubulin on cilia using confocal microscopy. Importantly, NTHi infection did not adversely affect normal ciliary function (CBF and percentage area of motile cilia) on non-PCD ALI-cultured airway cells over the experimental period. Taken together, we found the only difference between PCD and non-PCD was in ciliary motility, supporting the hypothesis that abnormal ciliary function in PCD increases the risk of NTHi biofilm development.

PCD patients exhibit abnormally low airway (particularly nasal) NO levels, the causes of which are still being debated [18]. Previously it was found that ALI-cultured PCD primary epithelial cells fail to increase NO levels early after infection with $S$. pneumoniae, suggesting that NO biosynthesis and iNOS induction was abnormal in PCD epithelial cells [20]. In contrast, the work herein extends our previous study and 


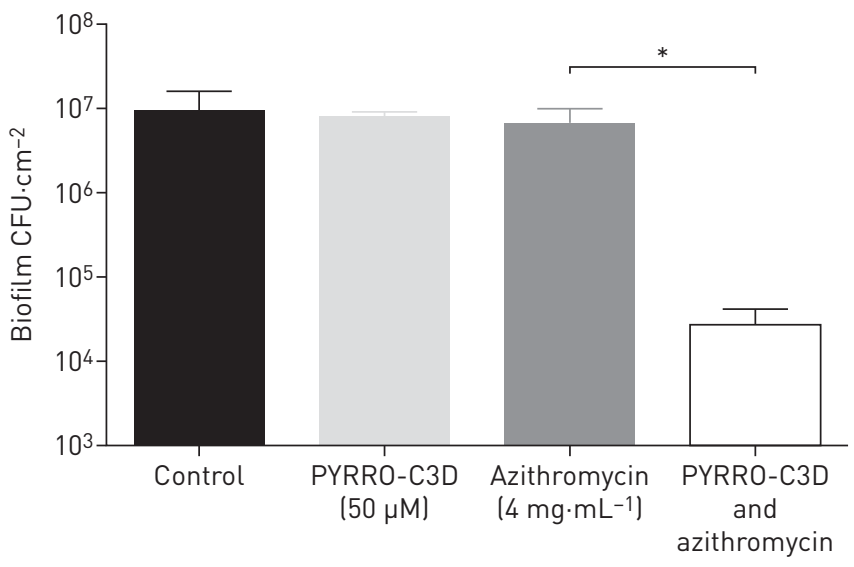

FIGURE 6 Treatment of primary ciliary dyskinesia (PCD) air-liquid interface (ALI)-differentiated airway epithelial cells with antibiotic and nitric oxide (NO) donor prodrug. The graph shows mean \pm SEM log biofilm colony forming unit counts (CFU $\mathrm{cm}^{-2}$ ) for adherent (biofilm) non-typeable Haemophilus influenzae (NTHi) co-cultured for $72 \mathrm{~h}$ on PCD airway epithelial cells that were cultured and ciliated at ALI ( $\mathrm{n}=4)$. Co-cultures were incubated for $2 \mathrm{~h}$, either without (control) or with NO donor prodrug PYRRO-C3D $(50 \mu \mathrm{M}), 4 \mathrm{mg} \cdot \mathrm{mL}^{-1}$ azithromycin, or both. NTHi viability was decreased nearly 3 logs by PYRRO-C3D/azithromycin treatment. $*$ : $p<0.05$.

demonstrates that NO biosynthesis is similar in PCD and non-PCD epithelial cells, both constitutively and following NTHi infection [22]. We have now also assessed NO levels using complementary methods and show that PCD and non-PCD cells exhibit similar NO biosynthesis and NOS isoenzyme expression that co-localises with the cilia marker $\beta$-tubulin, during co-culture. These studies support our previous data showing that PCD epithelial cells are capable of biosynthesising NO. Primary epithelial cell NO responses may, however, vary with the type of bacteria, and notably the PCD NTHi isolate did not induce the cytotoxic effects observed with $S$. pneumoniae [38].

Our results indicate that NTHi is present in matrix-enclosed aggregates adherent to polarised ciliated PCD epithelial cells [8]. Biofilm development is well known to reduce the efficacy of antibiotic therapy. Our data indicate that NTHi biofilms on airway epithelial cell cultures were tolerant of azithromycin treatment that easily kills planktonic NTHi [25], as it was ineffective in reducing NTHi viability. In contrast, co-administration of azithromycin with the novel pro-drug PYRRO-C3D, designed to target NO release specifically to bacteria following $\beta$-lactamase activation, reduced NTHi viability by nearly 3 logs on PCD epithelial cells. These results support recent studies with S. pneumoniae [28] and NTHi [25] showing that NO plays a role in modulating metabolic activity in these respiratory bacteria, resulting in increased antibiotic sensitivity. Importantly, our airway epithelial cell co-culture model permitted the translational investigation of anti-NTHi biofilm treatments in a physiologically relevant context with human primary cells. These results suggest that adjunctive exogenous NO therapy administered in combination with antibiotics to treat infections warrants further investigation.

This study has some limitations. First, because PCD cases are rare $(\approx 1: 10000)$, the co-culture experiments are challenging to replicate in high numbers. Secondly, we examined a limited number of clinical NTHi isolates in co-cultures. Thirdly, our studies focused on epithelial cells and did not investigate other innate cell responses that are important in host defences to NTHi, or may contribute to disease progression. Despite these limitations, our studies suggest that NTHi biofilms play a role in the chronicity of respiratory infections in PCD and provide hypothesis-driven research questions for further work assessing mechanisms of pathogenesis.

In summary, this study reports key observations regarding NTHi infection of primary ciliated epithelial cells from PCD patients. Firstly, abnormal ciliary function in PCD appears to be more permissive for NTHi biofilm development than non-PCD airway cells that have normal motility. Secondly, ALI-cultured primary airway cells from PCD patients appear to be competent in several defensive responses following NTHi infection, exhibiting comparable cytokine and LL-37 production with non-PCD airway cells. Thirdly, although nasal NO levels are abnormally low in most cases of PCD, ALI-cultured airway cells from PCD patients produced similar levels of NO following NTHi co-culture compared with non-PCD cells. Fourthly, this study demonstrates that our co-culture model provided a robust assay to investigate anti-infective treatments. Finally, we show that targeted exogenous NO enhanced antibiotic killing of NTHi in biofilms growing on PCD epithelia, suggesting a novel therapeutic approach for NTHi infection in PCD. 


\section{Acknowledgements}

We would like to thank Anton Page and Patricia Goggin, Biomedical Imaging Unit, University of Southampton, for their advice and help with electron microscopy techniques. We would also like to thank James Thompson and Janice Coles for their help and support with cell culturing and the patients who provided nasal brushing samples obtained from our Primary Ciliary Dyskinesia Centre, University Hospital Southampton NHS Foundation Trust, Southampton, UK.

\section{References}

1 Goutaki M, Meier AB, Halbeisen FS, et al. Clinical manifestations in primary ciliary dyskinesia: systematic review and meta-analysis. Eur Respir J 2016; 48: 1081-1095.

2 Alanin MC, Nielsen KG, von Buchwald C, et al. A longitudinal study of lung bacterial pathogens in patients with primary ciliary dyskinesia. Clin Microbiol Infect 2015; 21: 1093 e1091-1097.

3 Ngo CC, Massa HM, Thornton RB, et al. Predominant bacteria detected from the middle ear fluid of children experiencing otitis media: a systematic review. PLoS One 2016; 11: e0150949.

4 Greiner LL, Watanabe H, Phillips NJ, et al. Nontypeable Haemophilus influenzae strain 2019 produces a biofilm containing $\mathrm{N}$-acetylneuraminic acid that may mimic sialylated O-linked glycans. Infect Immun 2004; 72 : 4249-4260.

5 Jurcisek J, Greiner L, Watanabe $\mathrm{H}$, et al. Role of sialic acid and complex carbohydrate biosynthesis in biofilm formation by nontypeable Haemophilus influenzae in the chinchilla middle ear. Infect Immun 2005; 73: 3210-3218.

6 Hall-Stoodley L, Hu FZ, Gieseke A, et al. Direct detection of bacterial biofilms on the middle-ear mucosa of children with chronic otitis media. JAMA 2006; 296: 202-211.

$7 \quad$ Nistico L, Kreft R, Gieseke A, et al. Adenoid reservoir for pathogenic biofilm bacteria. J Clin Microbiol 2011; 49: $1411-1420$.

8 Hoiby N, Bjarnsholt T, Moser C, et al. Biofilms ESGf, consulting external expert Werner Z. ESCMID guideline for the diagnosis and treatment of biofilm infections 2014. Clin Microbiol Infect 2015; 21: Suppl. 1, S1-25.

9 Costerton JW, Stewart PS, Greenberg EP. Bacterial biofilms: a common cause of persistent infections. Science 1999; 284: $1318-1322$

10 Chole RA, Faddis BT. Anatomical evidence of microbial biofilms in tonsillar tissues: a possible mechanism to explain chronicity. Arch Otolaryngol Head Neck Surg 2003; 129: 634-636.

11 Starner TD, Zhang N, Kim G Jr, et al. Haemophilus influenzae forms biofilms on airway epithelia: implications in cystic fibrosis. Am J Respir Crit Care Med 2006; 174: 213-220.

12 Psaltis AJ, Ha KR, Beule AG, et al. Confocal scanning laser microscopy evidence of biofilms in patients with chronic rhinosinusitis. The Laryngoscope 2007; 117: 1302-1306.

13 Marsh RL, Thornton RB, Smith-Vaughan HC, et al. Detection of biofilm in bronchoalveolar lavage from children with non-cystic fibrosis bronchiectasis. Pediatr Pulmonol 2014.

14 Ciofu O, Tolker-Nielsen T, Jensen PO, et al. Antimicrobial resistance, respiratory tract infections and role of biofilms in lung infections in cystic fibrosis patients. Adv Drug Deliv Rev 2015; 85: 7-23.

15 Hall-Stoodley L, Stoodley P. Evolving concepts in biofilm infections. Cell Microbiol 2009; 11: 1034-1043.

16 Miyamoto N, Bakaletz LO. Kinetics of the ascension of NTHi from the nasopharynx to the middle ear coincident with adenovirus-induced compromise in the chinchilla. Microb Pathog 1997; 23: 119-126.

17 Pittet LA, Hall-Stoodley L, Rutkowski MR, et al. Influenza virus infection decreases tracheal mucociliary velocity and clearance of Streptococcus pneumoniae. Am J Respir Cell Mol Biol 2010; 42: 450-460.

18 Walker WT, Jackson CL, Lackie PM, et al. Nitric oxide in primary ciliary dyskinesia. Eur Respir J 2012; 40: $1024-1032$.

19 Collins SA, Gove K, Walker W, et al. Nasal nitric oxide screening for primary ciliary dyskinesia: systematic review and meta-analysis. Eur Respir J 2014; 44: 1589-1599.

20 Smith CM, Fadaee-Shohada MJ, Sawhney R, et al. Ciliated cultures from patients with primary ciliary dyskinesia do not produce nitric oxide or inducible nitric oxide synthase during early infection. Chest 2013; 144: 1671-1676.

21 Fowler CJ, Olivier KN, Leung JM, et al. Abnormal nasal nitric oxide production, ciliary beat frequency, and Toll-like receptor response in pulmonary nontuberculous mycobacterial disease epithelium. Am J Respir Crit Care Med 2013; 187: 1374-1381.

22 Walker WT, Jackson CL, Coles J, et al. Ciliated cultures from patients with primary ciliary dyskinesia produce nitric oxide in response to Haemophilus influenzae infection and proinflammatory cytokines. Chest 2014; 145: 668-669.

23 Barbato A, Frischer T, Kuehni CE, et al. Primary ciliary dyskinesia: a consensus statement on diagnostic and treatment approaches in children. Eur Respir J 2009; 34: 1264-1276.

24 Jackson CL, Behan L, Collins SA, et al. Accuracy of diagnostic testing in primary ciliary dyskinesia. Eur Respir J 2016; 47: 837-848.

25 Collins SA, Kelso MJ, Rineh A, et al. Cephalosporin-3'-diazeniumdiolate NO donor prodrug PYRRO-C3D enhances azithromycin susceptibility of nontypeable Haemophilus influenzae biofilms. Antimicrob Agents Chemother 2017; 61 .

26 Hall-Stoodley L, Nistico L, Sambanthamoorthy K, et al. Characterization of biofilm matrix, degradation by DNase treatment and evidence of capsule downregulation in Streptococcus pneumoniae clinical isolates. BMC Microbiol 2008; 8: 173 .

27 Erlandsen SL, Kristich CJ, Dunny GM, et al. High-resolution visualization of the microbial glycocalyx with low-voltage scanning electron microscopy: dependence on cationic dyes. J Histochem Cytochem 2004; 52: $1427-1435$

28 Allan RN, Morgan S, Brito-Mutunayagam S, et al. Low concentrations of nitric oxide modulate Streptococcus pneumoniae biofilm metabolism and antibiotic tolerance. Antimicrob Agents Chemother 2016; 60: 2456-2466.

29 Hirst RA, Jackson CL, Coles JL, et al. Culture of primary ciliary dyskinesia epithelial cells at air-liquid interface can alter ciliary phenotype but remains a robust and informative diagnostic aid. PloS one 2014; 9: e89675.

30 Ong HX, Jackson CL, Cole JL, et al. Primary air-liquid interface culture of nasal epithelium for nasal drug delivery. Mol Pharm 2016; 13: 2242-2252. 
31 Xiao C, Puddicombe SM, Field S, et al. Defective epithelial barrier function in asthma. J Allergy Clin Immunol 2011; 128: 549-556 e541-512.

32 Jackson CL, Lucas JS, Walker WT, et al. Neuronal NOS localises to human airway cilia. Nitric Oxide 2015; 44: $3-7$.

33 Djoba Siawaya JF, Roberts T, Babb C, et al. An evaluation of commercial fluorescent bead-based luminex cytokine assays. PLoS One 2008; 3: e2535.

34 Kojima H, Nakatsubo N, Kikuchi K, et al. Detection and imaging of nitric oxide with novel fluorescent indicators: diaminofluoresceins. Anal Chem 1998; 70: 2446-2453.

35 Cardines R, Giufre M, Pompilio A, et al. Haemophilus influenzae in children with cystic fibrosis: antimicrobial susceptibility, molecular epidemiology, distribution of adhesins and biofilm formation. Int J Med Microbiol 2012; 302: 45-52.

36 Bakaletz LO. Bacterial biofilms in the upper airway - evidence for role in pathology and implications for treatment of otitis media. Paediatr Respir Rev 2012; 13: 154-159.

37 Grainger CI, Greenwell LL, Lockley DJ, et al. Culture of Calu-3 cells at the air interface provides a representative model of the airway epithelial barrier. Pharm Res 2006; 23: 1482-1490.

38 Smith CM, Hirst RA, O'Callaghan C. In replay: ciliated cultures from patients with primary ciliary dyskinesia produce nitric oxide in response to Haemophilus influenzae infection and proinflammatory cytokines. Chest 2014; 145: 669 . 